\title{
On the Performance of Priority-Based Virtual Channels Scheduling Algorithm in Packet Telemetry System
}

\author{
TIAN Ye $\mathrm{e}^{1,2}$ \\ ${ }^{1}$ School of Information Science and Engineering, Shenyang Ligong University, Shenyang, China \\ ${ }^{2}$ State Key Laboratory of Rail Traffic Control and Safety (Beijing Jiaotong University), Beijing, China \\ Email: tianyereal@126.com \\ ZHANG Yan-qin* ${ }^{1}$ and ZHANG Zi-jing ${ }^{1}$ \\ ${ }^{1}$ School of Information Science and Engineering, Shenyang Ligong University, Shenyang, China \\ Email*: hnzyq2007@sina.com
}

\begin{abstract}
We Study the performance of priority-based virtual channels scheduling algorithm in packet telemetry system. Probability of occupying physical channel by the virtual channel with the highest priority is considered, on condition that the packet arrival rate contribution is Poisson distribution. Packets losing rate of the virtual channel with the highest priority is also investigated, of which calculating formulas are given. An interesting conclusion is made by theoretical analysis and simulation experiments that when the running time of the scheduling module is long enough, both the probability of being scheduled and packets losing rate of the virtual channel with the highest priority converge on fixed values, which can offer reference to engineering design.
\end{abstract}

Index Terms-priority; virtual channels; scheduling algorithm; packet telemetry

\section{INTRODUCTION}

With the development of space science and space technologies, some main worldwide space organizations established Consultive Committee for Space Data Systems(CCSDS) in 1982 to meet the demands of space missions[1]. Since then, CCSDS has designed a group of space communication standards, which are now widely used in more than 250 countries and regions[2-4]. Among these standards, TM Space Data Link protocol is a data communication and transmission protocol, which is mainly used(but not limited) in packet telemetry system to transmit packet telemetry data through the downlink, namely, from space to ground[5-7]. In order to transmit those information better, a two-layer multiplexing mechanism is used in packet telemetry system, which includes packet channel multiplexing mechanism and virtual channel multiplexing mechanism. Packet channel

Manuscript received April 6, 2010; revised June 1, 2008; accepted July 1, 2008.

*ZHANG Yan-qin, corresponding author. multiplexing mechanism makes a certain number of data packets share a virtual channel(VC), while virtual channel multiplexing mechanism makes a certain number of virtual channels share a physical channel.

Virtual channels are a group of logic channels formed by dividing the physical channel by different time slots. Each virtual channel transmits the packets information with the same or similar service demands, and the physical channel is shared by all these virtual channels. Thus the physical channel can be used to transmit various upper-layer sources data stream with different types and transmission requirements. The algorithm used in virtual channels mechanism, namely, virtual channels scheduling algorithm, determines the sequence of occupying the physical channel by each virtual channel, which has a great effect on the transmission delay and efficiency. In Packet Telemetry System, the scheduling algorithms often used include mainly fist-in-first-out algorithm, maximum remained prior algorithm, polling algorithm, priority-based algorithm. In some complicated systems, dynamic-priority-based algorithm may be used.

In this paper, we study the priority-based virtual channels scheduling algorithm in packet telemetry system. For this algorithm, each VC is assigned a priority number according to its importance and real-time requirements before the system begins to work. The important the VC is, or the higher the real-time required by the $\mathrm{VC}$ is, the higher the priority assigned will be. At each scheduling time point, the one with the highest priority among the VCS whose frames buffer does exist frames can be scheduled, and the first frame in its buffer is transmitted through the physical channel(namely, the VC occupies the physical channel). Through theoretical analysis and simulation results, we find an interesting conclusion: when the running time of the scheduling module is long enough, for the $\mathrm{VC}$ with the highest priority, both the probability of being scheduled and the packets losing rate converge on fixed values, which can provide reference for engineering design. 


\section{SYSTEM MODEL}

In this paper, we choose the packet telemetry source model, of which the physical channel is divided into 4 virtual channels to transfer all user data through the downlink[8], as illustrated in Fig.1. The 4 virtual channels are shown as follows:

$\mathrm{VC} 1$ : transmitting general engineering data, with the second higher priority.

VC2: transmitting important express data, with the highest priority.

VC3: transmitting download data, with the lowest priority.

VC4: producing idle frames. At any scheduling time point, if there are no frames in all the other VCs buffers, an idle frame is generated in VC4 buffer and transmitted through the physical channel.
For $\mathrm{VCi}, \mathrm{i}=1,2,3$, the packet arriving process is Poisson distribution with the parameter $\lambda_{1}$, and the packet length is a fixed value[9]. Let $I_{p i}$ be the Packet length, and $I_{m p}$ be the MPDU packet zone length of the frame, then each frame contains $N_{i}=I_{m p} / I_{p i}$ packets, which also means that a frame is generated and sent to the VCi buffer at the arriving time of the $\mathrm{mN}_{\mathrm{i}}$ packet, $\mathrm{m}=1,2,3 \mathrm{~L}$.

At each scheduling time point, the scheduling module choose the one with the highest priority among the VCs whose frame buffers are not empty, and transmit the first frame in its buffer through the physical channel. If buffers of VC1, VC2, VC3 are all empty, an idle frame is generated in VC4 buffer and transmitted

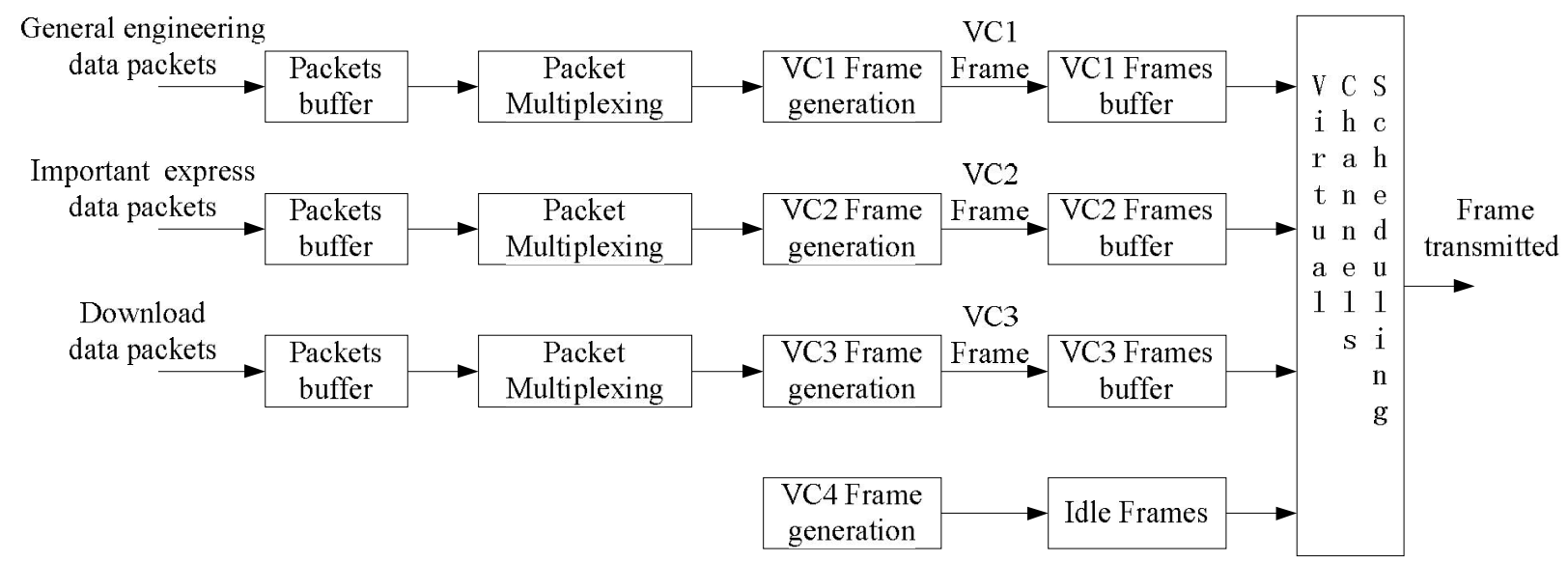

Figure 1. Packet Telemetry Source Model

\section{THEORETICAL ANAYSIS}

\section{A. the size of packets buffer is infinte}

First consider the condition that the size of packets buffer is infinite. At the first scheduling time point, $\Delta t$, if there is one frame or more in the VC2 frames buffer, which means the number of packets arriving in the packets buffer during the time interval $[0, \Delta \mathrm{t}]$ is larger than or equal to $\mathrm{N}_{2}, \mathrm{VC} 2$ can be scheduled. So we can get the probability of $\mathrm{VC} 2$ can be scheduled at the time $\Delta t, P_{\mathrm{VC}_{2}}(1)$, as

$$
\begin{aligned}
P_{\mathrm{IVC} 2}(1) & =P\left(A_{\mathrm{VC}_{2}}(\Delta t) \geq N_{2}\right) \\
& =\sum_{n=N_{2}}^{\infty} P\left(A_{\mathrm{VC}_{2}}(\Delta t)=n\right)
\end{aligned}
$$

where $A_{v c_{2}}(\Delta t)$ means the number of packets arriving in $\mathrm{VC} 2$ during the time interval $[0, \Delta \mathrm{t}]$. For the packet arriving process which obeys Poisson distribution, the probability of arriving $n$ packets during the time period $\Delta \mathrm{t}, \mathrm{P}\left(\mathrm{A}_{\mathrm{vc} 2}(\Delta \mathrm{t})=\mathrm{n}\right)$, can be calculated as:

$$
P\left(A_{V C_{2}}(\Delta t)=n\right)=\frac{\left(\lambda_{2} \Delta t\right)^{n} e^{-\lambda_{2} \Delta t}}{n !}
$$

Substituting equation (2) into equation (1), we can get

$$
P_{I V C 2}(1)=\sum_{n=N_{2}}^{\infty} \frac{\left(\lambda_{2} \Delta t\right)^{n} e^{-\lambda_{2} \Delta t}}{n !}
$$

Otherwise, if the number of packets arriving in the packets buffer during the time interval $[0, \Delta \mathrm{t}]$ is less than $\mathrm{N}_{2}$, there will be no frames in VC2 buffer at the time $\Delta \mathrm{t}$, and VC2 cannot be scheduled. So we can get the probability of $\mathrm{VC} 2$ can be scheduled at the time $\Delta \mathrm{t}$, $\mathrm{P}_{\text {IVC } 2}(0)$, as

$$
\begin{aligned}
P_{\mathrm{IVC} 2}(0) & =P\left(A_{V_{2} 2}(\Delta t)<N_{2}\right) \\
& =\sum_{n=0}^{N_{2}-1} P\left(A_{v C_{2}}(\Delta t)=n\right)
\end{aligned}
$$

Substituting equation (2) into equation (4), we can get

$$
P_{\text {IVC } 2}(0)=\sum_{n=0}^{N_{2}-1} \frac{\left(\lambda_{2} \Delta t\right)^{n} e^{-\lambda_{2} \Delta t}}{n !}
$$

For the size of packets buffer is infinite, there will be no packets lost. The packets not transmitted at the time $\Delta t$ will be stored in the packets buffer. Let $R_{11}$ be the number of packets remained in VC2 packets buffer after the time $\Delta t, P\left(R_{11}=r\right)$ be the probability of $R_{11}=r$. If 
$\mathrm{r}=0,1,2, \mathrm{~L} \mathrm{~N}-1, \mathrm{r}$ packets remained means that during the time interval $[0, \Delta t]$, there are $\mathrm{N}_{2}+r$ packets arriving(in this case, $\mathrm{VC} 2$ is scheduled, $\mathrm{N}_{2}$ packets transmitted and $r$ packets remained) or $r$ packets arriving (in this case, $\mathrm{VC} 2$ is not scheduled, all of the $r$ packets remained ), so we have

$$
\begin{aligned}
P\left(R_{11}=r\right)=P\left(A_{v C_{2}}(\Delta t)=N_{2}+r\right) & +P\left(A_{v C_{2}}(\Delta t)=r\right) \\
r & =0,1, L, N_{2}-1(6)
\end{aligned}
$$

Substituting equation (2) into equation (6), we can get

$$
P\left(R_{11}=r\right)=\frac{\left(\lambda_{2} \Delta t\right)^{N+r} e^{-\lambda_{2} \Delta t}}{\left(N_{2}+r\right) !}+\frac{\left(\lambda_{2} \Delta t\right)^{r} e^{-\lambda_{2} \Delta t}}{r !}
$$

$$
\begin{gathered}
r=0,1, L, N_{2}-1(7) \\
P\left(R_{11}=r\right)= \begin{cases}\frac{\left(\lambda_{2} \Delta t\right)^{N_{2}+r} e^{-\lambda_{2} \Delta t}}{\left(N_{2}+r\right) !}+\frac{\left(\lambda_{2} \Delta t\right)^{r} e^{-\lambda_{2} \Delta t}}{r !} & r=0,1, L N_{2}-1 \\
\frac{\left(\lambda_{2} \Delta t\right)^{N_{2}+r} e^{-\lambda_{2} \Delta t}}{\left(N_{2}+r\right) !} & r=N_{2}, N_{2}+1, L, \infty\end{cases}
\end{gathered}
$$

At the second scheduling time point, $2 \Delta t$, if the total number of the packets remained at the time $\Delta t, R_{11}$, and the new packets arriving in the time interval $[\Delta t, 2 \Delta t], A_{v c_{2}}(\Delta t)$, is larger than or equal to $N_{2}$, there will be at least one frame in the VC2 frames buffer, and VC2 can be scheduled. Otherwise VC2 cannot be scheduled. Thus we can get the probability of VC2 can be scheduled at the time $2 \Delta \mathrm{t}, \mathrm{P}_{\mathrm{IVC}_{2}}{ }(1)$, as

$$
\begin{aligned}
P_{\mathrm{IVC} 2}{ }^{\prime \prime}(1) & =P\left(R_{11}+A_{v C_{2}}(\Delta t) \geq N_{2}\right) \\
& =\sum_{q=N_{2}}^{\infty} \sum_{r=0}^{q} P\left(R_{11}=r, A_{v C_{2}}(\Delta t)=q-r\right)
\end{aligned}
$$

For Poisson distribution, $R_{11}$ and $A_{v c_{2}}(\Delta t)$ are independent of each other, so we have

$$
\begin{aligned}
& P_{\text {IVC2 }}{ }^{\prime \prime}(1)=\sum_{q=N_{2}}^{\infty} \sum_{r=0}^{q} P\left(R_{11}=r, A_{V C 2}(\Delta t)=q-r\right) \\
& =\sum_{q=N_{2}}^{\infty} \sum_{r=0}^{q} P\left(R_{l 1}=r\right) \cdot P\left(A_{v C 2}(\Delta t)=q-r\right)
\end{aligned}
$$

If $r=N_{2}, N_{2}+1, L \infty, r$ packets remained means that during the time interval $[0, \Delta t]$, there are $\mathrm{N}_{2}+r$ packets arriving(VC2 is scheduled, $\mathrm{N}_{2}$ packets transmitted and $r$ packets remained), so we have

$$
\begin{aligned}
P\left(R_{11}=r\right) & =P\left(A_{v c_{2}}(\Delta t)=N_{2}+r\right) \\
& =\frac{\left(\lambda_{2} \Delta t\right)^{N+r} e^{-\lambda_{2} \Delta t}}{\left(N_{2}+r\right) !}
\end{aligned}
$$

Combing equation (7) and (8), $P\left(R_{11}=r\right)$ can be expressed as
The probability of VC2 cannot be scheduled at the time $2 \Delta \mathrm{t}, \mathrm{P}_{\mathrm{IVC} 2} "(0)$, can be calculated as

$$
\begin{aligned}
P_{I V C 2} "(0) & =\sum_{q=0}^{N_{2}-1} \sum_{r=0}^{q} P\left(R_{11}=r, A_{V C 2}(\Delta t)=q-r\right) \\
& =\sum_{q=0}^{N_{2}-1} \sum_{r=0}^{q} P\left(R_{11}=r\right) \cdot P\left(A_{V C 2}(\Delta t)=q-r\right)
\end{aligned}
$$

Let $R_{12}$ be the number of packets remained in VC2 packets buffer after the time $2 \Delta t, P\left(R_{12}=r\right)$ be the probability of $R_{12}=r$.If $r=0,1,2, L N-1, r$ packets remained means that the total number of the packets remained at the time $\Delta t, R_{11}$, and the new packets arriving in the time interval $[\Delta t, 2 \Delta t], A_{v c_{2}}(\Delta t)$, is $N_{2}+r$ (in this case, $\mathrm{VC} 2$ is scheduled at the time $2 \Delta \mathrm{t}, \mathrm{N}_{2}$ packets transmitted and $r$ packets remained) or $r$ (in this case, VC2 is not scheduled at the time $2 \Delta t$, all of the $r$ packets remained ), so we have

where the values of $P\left(A_{v c_{2}}(\Delta t)=q-r\right)$ and $P\left(R_{11}=r\right)$ can be calculated by using equation (2) and equation (9), respectively.

$$
\begin{aligned}
P\left(R_{12}=r\right) & =P\left(R_{11}+A_{v c 2}(\Delta t)=N_{2}+r\right)+P\left(R_{11}+A_{v c 2}(\Delta t)=r\right) \\
& =\sum_{q=0}^{N_{2}+r} P\left(R_{11}=q, A_{v c 2}(\Delta t)=N_{2}+r-q\right)+\sum_{q=0}^{r} P\left(R_{11}=q, A_{v c 2}(\Delta t)=r-q\right) \\
& =\sum_{q=0}^{N_{2}+r} P\left(R_{11}=q\right) \cdot P\left(A_{v c 2}(\Delta t)=N_{2}+r-q\right)+\sum_{q=0}^{r} P\left(R_{11}=q\right) \cdot P\left(A_{v c 2}(\Delta t)=r-q\right)
\end{aligned}
$$

$$
\mathrm{r}=0,1, \mathrm{~L}, \mathrm{~N}_{2}-1
$$


If $\mathrm{r}=\mathrm{N}_{2}, \mathrm{~N}_{2}+1, \mathrm{~L} \infty, \mathrm{r}$ packets remained means that the total number of the packets remained at the time $\Delta t, R_{11}$, and the new packets arriving in the time interval $[\Delta t, 2 \Delta t], A_{v{ }_{2}}(\Delta t)$, is $N_{2}+r(V C 2$ is scheduled at the time $2 \Delta \mathrm{t}, \mathrm{N}_{2}$ packets transmitted and $\mathrm{r}$ packets remained), so we have

$$
\begin{aligned}
P\left(R_{12}=r\right) & =P\left(R_{11}+A_{v c 2}(\Delta t)=N_{2}+r\right) \\
= & \sum_{q=0}^{N_{2}+r} P\left(R_{11}=q, A_{v c 2}(\Delta t)=N_{2}+r-q\right) \\
= & \sum_{q=0}^{N_{2}+r} P\left(R_{11}=q\right) \cdot P\left(A_{v c 2}(\Delta t)=N_{2}+r-q\right) \\
r & =N_{2}, N_{2}+1, L \infty
\end{aligned}
$$

Similarly, at the kth scheduling time point, $k \Delta t$, if the total number of the remained packets at the time $(\mathrm{k}-1) \Delta \mathrm{t}, \mathrm{R}_{\mathrm{lk}-1}$, and the number of packets arriving during the time period $[(\mathrm{k}-1) \Delta \mathrm{t}, \mathrm{k} \Delta \mathrm{t}], \mathrm{A}_{\mathrm{v} \mathrm{C}_{2}}(\Delta \mathrm{t})$, is larger than or equal to $\mathrm{N}_{2}$, there will be at least one frame in the VC2 frames buffer. Thus VC2 can be scheduled. Otherwise VC2 cannot be scheduled. So at the time $k \Delta t$, the probability of VC2 can be scheduled, $\mathrm{P}_{\mathrm{IVC} 2}{ }^{(\mathrm{k})}(1)$, and the probability of VC2 cannot be scheduled, $\mathrm{P}_{\mathrm{IVC} 2}{ }^{(\mathrm{k})}(0)$, can be expressed as

$$
\begin{aligned}
& P_{\mathrm{IVC} 2}{ }^{(k)}(1)=P\left(R_{\mathrm{Ik}-1}+\mathrm{A}_{\mathrm{VC} 2}(\Delta \mathrm{t}) \geq \mathrm{N}_{2}\right) \\
& =\sum_{q=N_{2}}^{\infty} \sum_{r=0}^{q} P\left(R_{I k-1}=r, A_{v C_{2}}(\Delta t)=q-r\right) \\
& =\sum_{\mathrm{q}=\mathrm{N}_{2}}^{\infty} \sum_{\mathrm{r}=0}^{\mathrm{q}} \mathrm{P}\left(\mathrm{R}_{\mathrm{lk}-1}=\mathrm{r}\right) \cdot \mathrm{P}\left(\mathrm{A}_{\mathrm{vc} 2}(\Delta \mathrm{t})=\mathrm{q}-\mathrm{r}\right) \\
& P\left(R_{1 k}=r\right)=\left\{\begin{array}{l}
\sum_{q=0}^{N_{2}+r} P\left(R_{1 k-1}=q\right) \cdot P\left(A_{v c_{2}}(\Delta t)=N_{2}+r-q\right)+\sum_{q=0}^{r} P\left(R_{l k-1}=q\right) \cdot P\left(A_{v c 2}(\Delta t)=r-q\right), r=0,1, L N_{2}-1 \\
\sum_{q=0}^{N_{2}+r} P\left(R_{\mid k-1}=q\right) \cdot P\left(A_{v c_{2}}(\Delta t)=N_{2}+r-q\right), \quad r=N_{2}, N_{2}+1, L \infty
\end{array}\right.
\end{aligned}
$$

$$
\begin{aligned}
\mathrm{P}_{\mathrm{IVC} 2}{ }^{(\mathrm{k})}(0) & =\mathrm{P}\left(\mathrm{R}_{\mathrm{Ik}-1}+\mathrm{A}_{\mathrm{vC} 2}(\Delta \mathrm{t})<\mathrm{N}_{2}\right) \\
& =\sum_{\mathrm{q}=0}^{\mathrm{N}_{2}-1} \sum_{\mathrm{r}=0}^{\mathrm{q}} \mathrm{P}\left(\mathrm{R}_{\mathrm{Ik}-1}=r, A_{\mathrm{vC} 2}(\Delta \mathrm{t})=\mathrm{q}-\mathrm{r}\right) \\
& =\sum_{\mathrm{q}=0}^{\mathrm{N}_{2}-1} \sum_{\mathrm{r}=0}^{\mathrm{q}} \mathrm{P}\left(\mathrm{R}_{\mathrm{Ik}-1}=\mathrm{r}\right) \cdot \mathrm{P}\left(\mathrm{A}_{\mathrm{vc} 2}(\Delta \mathrm{t})=\mathrm{q}-\mathrm{r}\right)
\end{aligned}
$$

$$
\mathrm{k}=3,4 \mathrm{~L} \infty
$$

Let $R_{I k}$ be the number of packets remained in VC2 packets buffer after the time $k \Delta t, P\left(R_{I k}=r\right)$ be the

$$
\mathrm{k}=3,4 \mathrm{~L} \infty
$$

\section{B. the size of packets buffer is finite}

In practical application, the size of packets buffer is often finite. In this case, the performance of the scheduling algorithm is different from that of the analysis above.

At the first scheduling time point, $\Delta t$, if the number of packets arriving in VC2 packets buffer is larger than $\mathrm{N}_{2}$, then there will be at least one frame in VC2 frames buffer, which means VC2 can occupy physical channel. So we have the probability of VC2 occupying physical channel at the time $\Delta \mathrm{t}, \mathrm{P}_{\mathrm{vC} 2}(1)$, as

$$
P_{\mathrm{VC}_{2}}(1)=P\left(A_{\mathrm{VC}_{2}}(\Delta t) \geq N_{2}\right)=\sum_{n=N_{2}}^{\infty} \frac{\left(\lambda_{2} \Delta t\right)^{n} e^{-\lambda_{2} \Delta t}}{n !}
$$

where $A_{v c_{2}}(\Delta t)$ means the number of packets arriving in VC2 during the time interval $[0, \Delta \mathrm{t}]$, whose value can be calculated by equation (2).

Otherwise, there will be no frames in VC2 frames buffer, which means VC2 can not occupy physical 
channel. So we have the probability of VC2 not occupying physical channel at the time $\Delta \mathrm{t}, \mathrm{P}_{\mathrm{vc} 2}(0)$, as

$$
P_{v_{2} 2}(0)=P\left(A_{v c_{2}}(\Delta t)<N_{2}\right)=\sum_{n=0}^{N_{2}-1} \frac{\left(\lambda_{2} \Delta t\right)^{n} e^{-\lambda_{2} \Delta t}}{n !}
$$

Let $\mathrm{M}$ be the capacity of the VC2 packets buffer, probability of losing $d$ packets of $\mathrm{VC} 2$ at the time $\Delta \mathrm{t}, \mathrm{P}_{\mathrm{IVC} 2}^{\prime}(\mathrm{d})$, can be expressed as:

$$
P_{\text {IVC } 2}^{\prime}(d)=\left\{\begin{array}{lc}
\sum_{n=0}^{M} \frac{e^{-\lambda_{2} \Delta t}\left(\lambda_{2} \Delta t\right)^{n}}{n !} & d=0 \\
\frac{e^{-\lambda_{2} \Delta t}\left(\lambda_{2} \Delta t\right)^{M+d}}{(M+d) !} & d=1,2, L, \infty
\end{array}\right.
$$

After the first scheduling time point, probability of $r$ packets remained in $\mathrm{VC} 2$ packets buffer, $\mathrm{P}_{\mathrm{R}_{\mathrm{VC} 2}}(r)$, is

$$
\begin{aligned}
& P_{R_{\mathrm{vC} 2}}(r)=\left\{\begin{array}{cc}
\frac{\left(\lambda_{2} \Delta t\right)^{N_{2}+r} e^{-\lambda_{2} \Delta t}}{\left(N_{2}+r\right) !}+\frac{\left(\lambda_{2} \Delta t\right)^{r} e^{-\lambda_{2} \Delta t}}{r !} & r=0,1, L, M-N_{2}-1 \\
\sum_{i=M}^{\infty} \frac{\left(\lambda_{2} \Delta t\right)^{i} e^{-\lambda_{2} \Delta t}}{i !}+\frac{\left(\lambda_{2} \Delta t\right)^{M-N_{2}} e^{-\lambda_{2} \Delta t}}{\left(M-N_{2}\right) !} & r=M-N_{2} \\
\frac{\left(\lambda_{2} \Delta t\right)^{r} e^{-\lambda_{2} \Delta t}}{r !} & M-N_{2}<r<N_{2} \\
0 & \text { others }
\end{array}\right. \\
& P_{R_{\mathrm{Vc} 2}}(r)=\left\{\begin{array}{cc}
\frac{\left(\lambda_{2} \Delta t\right)^{N_{2}+r} e^{-\lambda_{2} \Delta t}}{\left(N_{2}+r\right) !}+\frac{\left(\lambda_{2} \Delta t\right)^{r} e^{-\lambda_{2} \Delta t}}{r !} & r=0,1, L, N_{2}-1 \\
\frac{\left(\lambda_{2} \Delta t\right)^{N_{2}+r} e^{-\lambda_{2} \Delta t}}{\left(N_{2}+r\right) !} & N_{2}-1<r<M-N_{2} \\
\sum_{i=M}^{\infty} \frac{\left(\lambda_{2} \Delta t\right)^{i} e^{-\lambda_{2} \Delta t}}{i !} & r=M-N_{2} \\
0 & \text { others }
\end{array}\right.
\end{aligned}
$$$$
\text { if } \mathrm{N}_{2}<\mathrm{M}<2 \mathrm{~N}_{2}
$$

if $M \geq 2 \mathrm{~N}_{2}$

Similarly, at the kth scheduling time point, $k \Delta t$, $\mathrm{k}=2,3 \mathrm{~L}$, when the total number of the packets remained after the time $(k-1) \Delta t, R_{\mathrm{vc} 2}((k-1) \Delta t)$, and the packets arriving during the time interval $[(k-1) \Delta t, k \Delta t], A_{v c 2}((k-1) \Delta t, k \Delta t)$, is larger than or equal to $\mathrm{N}_{2}$, there will be at least one frame in $\mathrm{VC} 2$ frames buffer. In this case, VC2 can be scheduled and the first frame in its buffer is transmitted through the physical channel, otherwise it cannot be scheduled. Thus we can get

$$
\begin{aligned}
P_{v C 2}{ }^{(k)}(1) & =P\left(\left[R_{v C_{2}}((k-1) \Delta t)+A_{v C_{2}}((k-1) \Delta t, k \Delta t)\right] \geq N_{2}\right) \\
& =\sum_{q=N_{2}}^{\infty} \sum_{i=0}^{q} P_{\mathrm{RV}{ }}{ }^{(k-1)}(\mathrm{i}) \mathrm{P}(\mathrm{A}(\Delta \mathrm{t})=\mathrm{q}-\mathrm{i})
\end{aligned}
$$

$$
\begin{aligned}
\mathrm{P}_{\mathrm{vc} 2}{ }^{(\mathrm{k})}(0) & =\mathrm{P}\left(\left[\mathrm{R}_{\mathrm{vc} 2}((\mathrm{k}-1) \Delta \mathrm{t})+\mathrm{A}_{\mathrm{vc} 2}((\mathrm{k}-1) \Delta \mathrm{t}, \mathrm{k} \Delta \mathrm{t})\right]<\mathrm{N}_{2}\right) \\
& =\sum_{\mathrm{q}=0}^{\mathrm{N}_{2}-1} \sum_{\mathrm{i}=0}^{\mathrm{q}} \mathrm{P}_{\mathrm{R}_{\mathrm{vC} 2}}{ }^{(\mathrm{k}-1)}(\mathrm{i}) \mathrm{P}\left(\mathrm{A}_{\mathrm{vc} 2}(\Delta \mathrm{t})=\mathrm{q}-\mathrm{i}\right)
\end{aligned}
$$

where $P_{\mathrm{VC} 2}{ }^{(k)}(1)$ and $P_{\mathrm{VC} 2}{ }^{(k)}(0)$ are the probability of VC2 can be scheduled and cannot be scheduled at the time $\mathrm{k} \Delta \mathrm{t}$, respectively.

Probability of losing $d$ packets of VC2 at the time $\mathrm{k} \Delta \mathrm{t}$ can be expressed as: 


$$
P^{(k)} \operatorname{vvc}_{2}(d)=\left\{\begin{array}{lc}
\sum_{q=0}^{M} \sum_{i=0}^{q} P_{R_{v c 2}}{ }^{(k-1)}(i) P\left(A_{v c 2}(\Delta t)=q-i\right) & d=0 \\
\sum_{q=0}^{M+d} P_{R_{v c 2}}(k-1)(q) P\left(A_{v C 2}(\Delta t)=M+d-q\right) & d=1,2, L \infty
\end{array}\right.
$$

After the kth scheduling time point, probability of $r$ packets remained in $\mathrm{VC} 2$ packets buffer is

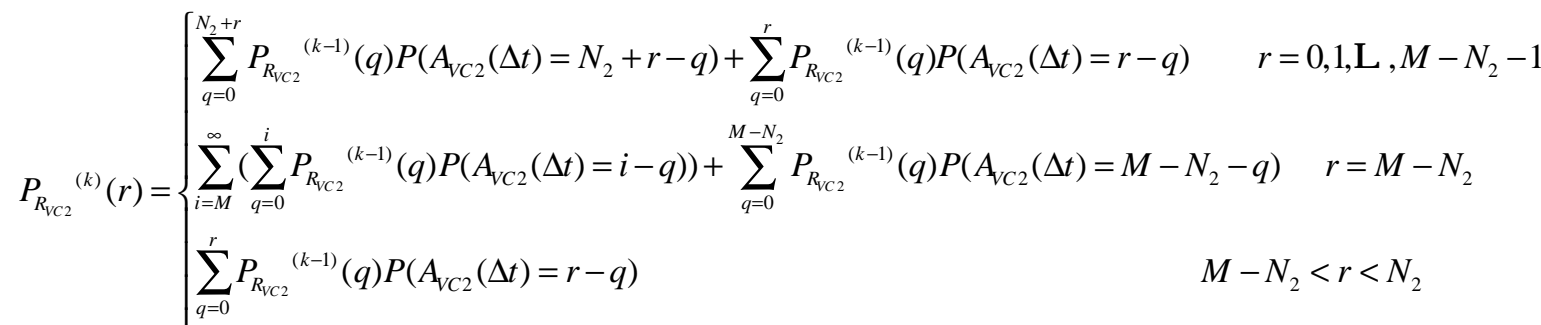

$$
\begin{aligned}
& 0 \text { others }
\end{aligned}
$$

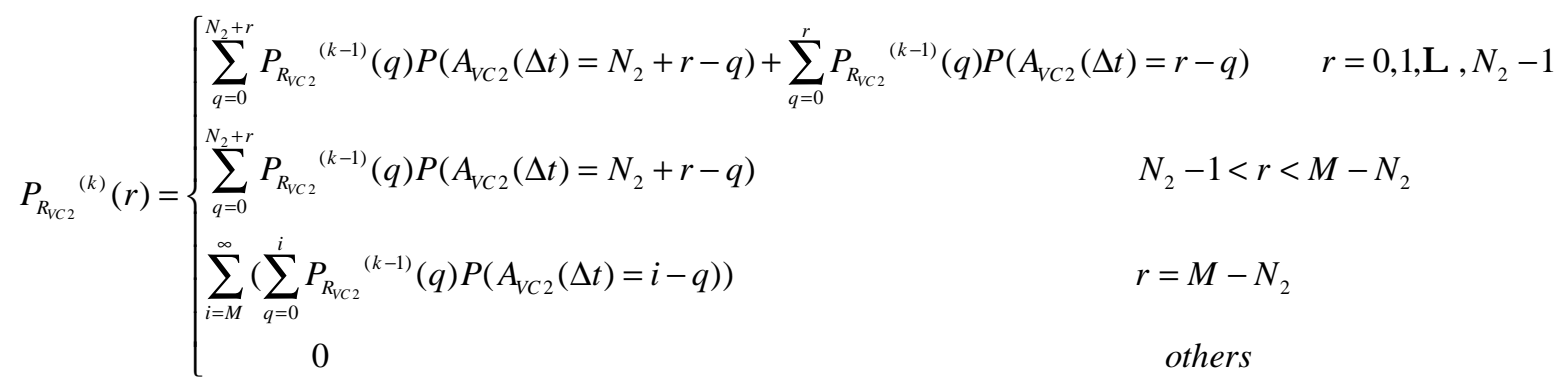

\section{SimULATION RESULTS}

Simulation parameters are set as follows:

(1) Source data rate of VC1, VC2 and VC3 are taken 10,10, 35 Mbps, respectively;

(2) The MPDU packet zone length of frame, $I_{m p}$, is 10000bits;

(3) The packet length of $\mathrm{VC} 1, \mathrm{VC} 2$ and $\mathrm{VC} 3$ are 1000, 2000, 500bits, respectively;

(4) The average packet arrival rate of $\mathrm{VC} 1, \mathrm{VC} 2$ andVC3 are 10000, 5000, 70000/s, respectively;

(5) The total simulation time $\mathrm{T}$ is $0.8 \mathrm{~s}$.

Fig.2 illustrates the probability that VC2 is scheduled at different scheduling time points.

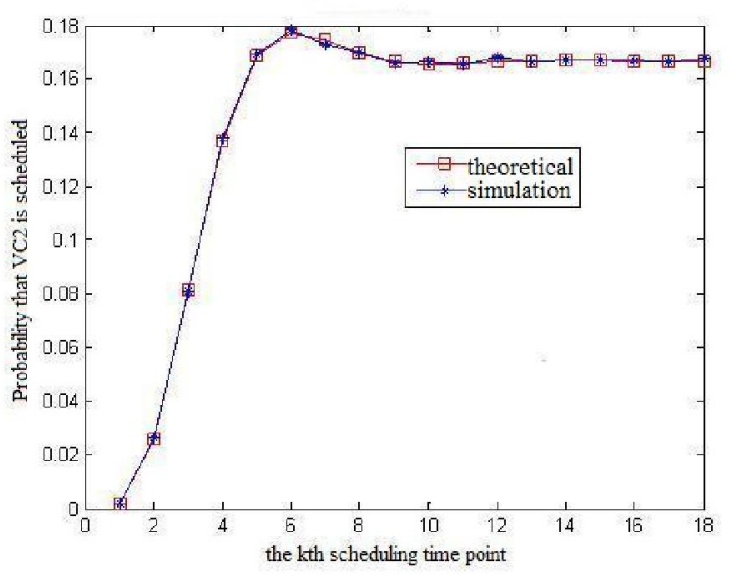

(a) $M$ is infinite 


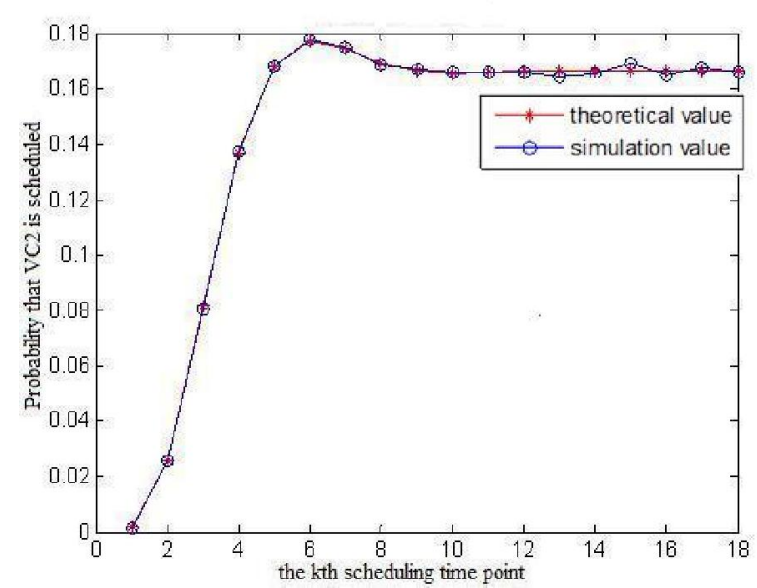

(b) $\mathrm{N}_{2}<\mathrm{M}<2 \mathrm{~N}_{2}\left(\mathrm{M}=8, \mathrm{~N}_{2}=5\right)$

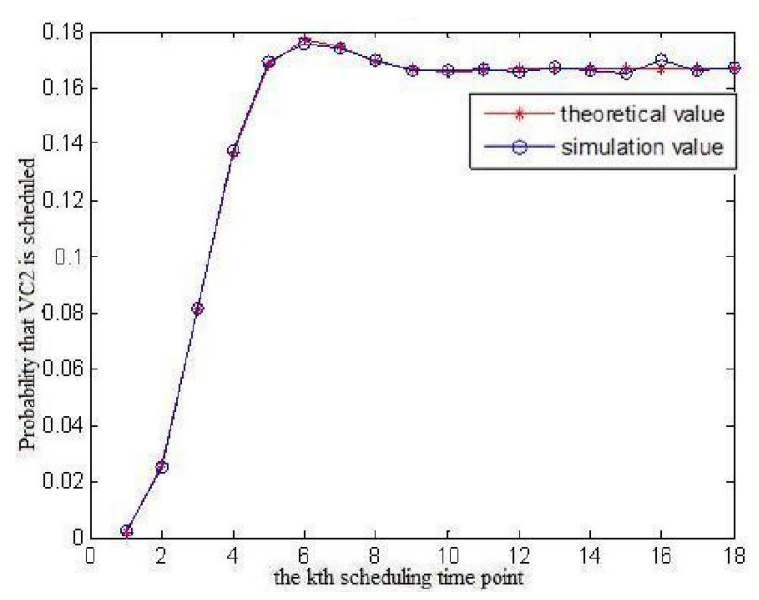

(c) $M \geq 2 N_{2}\left(M=15, N_{2}=5\right)$

Figure 2. Probability that VC2 is scheduled at different scheduling time points

From the simulation results we can see that with the increasing of the running time of the scheduling module, the probability that $\mathrm{VC} 2$ can be scheduled converges on a fixed value, and the simulation curve almost completely coincide with the theoretical curve, which proves the correctness of the analysis in section III.

Fig. 3 illustrates the curve of the theoretical value and simulation value of the packets losing rate of $\mathrm{VC} 2$ at different scheduling time points when the packet buffer capacity is $M(M=8)$, which proves the correctness of equation(26). In practical application, one can design the value of $\mathrm{M}$ according to the desired packets losing rate.

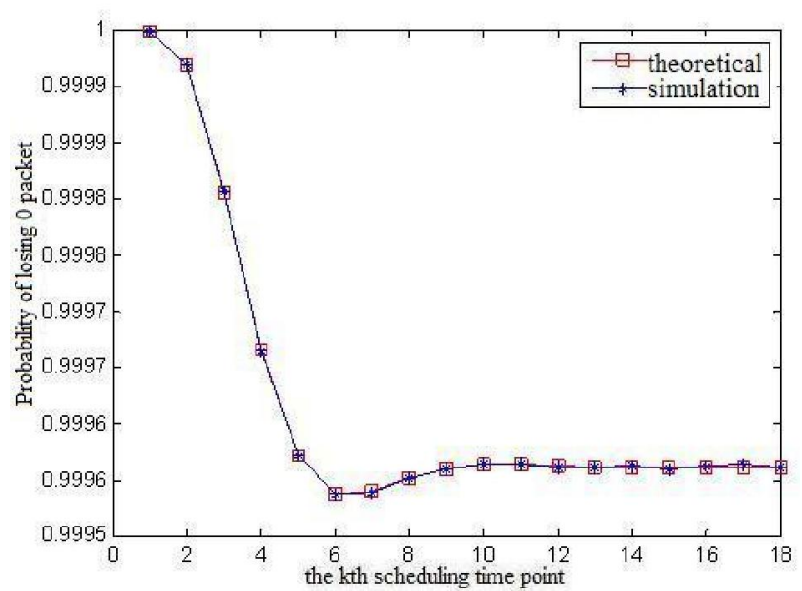

(a) Probability of losing 0 packet

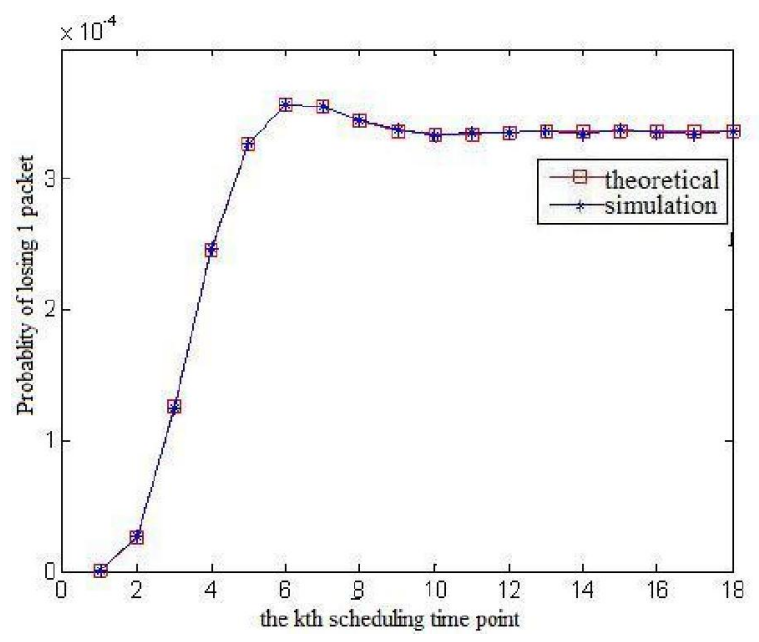

(b) Probability of losing 1 packet

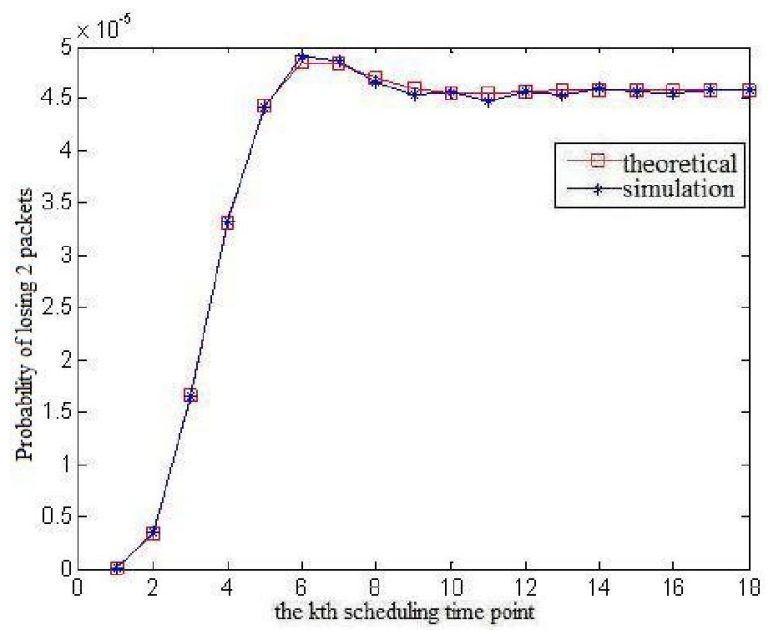

(c) Probability of losing 2 packets 


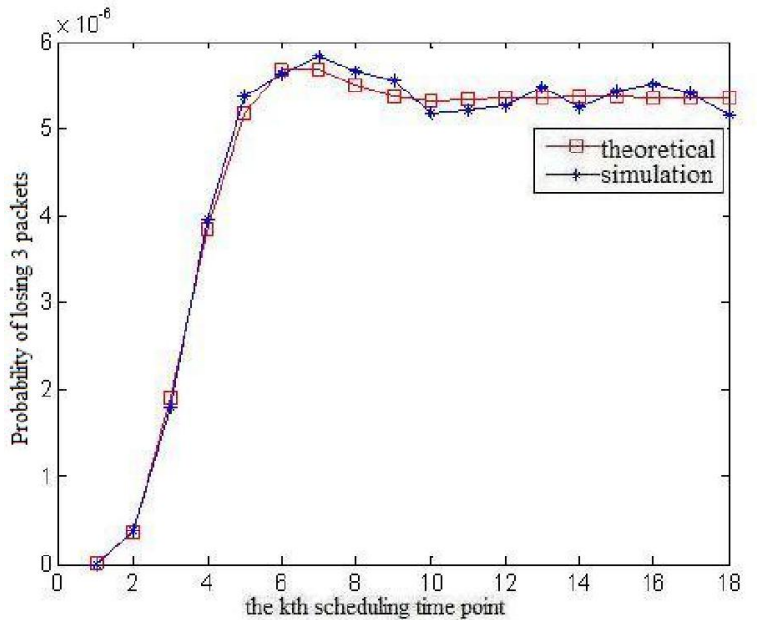

(d) Probability of losing 3 packets

Figure.3 Packets losing rate of VC2 at different scheduling time point

\section{CONCLUSION}

We study the scheduling algorithm based on priority in packet telemetry system. Through severe reasoning and simulation experiment, we find that when the running time of the scheduling module is long enough, the probability that the VC with the highest priority can be scheduled converges on a fixed value. We also propose the method to calculate packets losing rating of the VC with the highest priority, which can offer reference to engineering design.

\section{ACKNOWLEDGMENT}

This work was supported by the State Key Laboratory of Rail Traffic Control and Safety(RCS2009K008), Beijing Jiaotong University and Doctorial Start-up Foundation for Scientific Research of Liaoning Province(20101092).

\section{REFERENCES}

[1] CCSDS. Welcome to CCSDS.org. http: //public. ccsds. org/default.aspx. 2011.

[2] CCSDS. CCSDS missions. http: //public.ccsds.org/ implementations/ missions.aspx. 2007.

[3] Zhao He-ping, Li Ning-ning. Implementation of CCSDS standard in military space mission[J]. Spacecraft Engineering, 2007, 16(4):78-82.

[4] Zhou Jun. The study and simulation of space data link protocol based on CCSDS[D]. Changsha, China: National University of Defense Technology, 2007.

[5] CCSDS. Space packet protocol[S]. CCSDS 133.0-B-1, Blue Book, September 2003.

[6] CCSDS. TM space data link protocol[S]. CCSDS 132.0B-1, Blue Book, September 2003.

[7] CCSDS. TM synchronization and Channel Coding[S]. CCSDS 131.0-B-1, Blue Book, September 2003.

[8] Gu Ying-qi, Tan Wei-chi. CCSDS downlink virtual channel schedule and performance analysis[J]. Chinese Space Science and Technology, 2001, 21(3):29-35.

[9] Ba Yong, Zhang Nai-tong. Analysis of CCSDS protocol and space data system[D]. Harbin: Harbin Institute of Technology, 2000.

[10] Mao Yong-cai, Hu Qi-ying. Stochastic Process[M]. Xi'an, China : Xidian University Press. 2006:39-45.

TIAN Ye, born in 1977, received Doctor's degree of Communication and Information Systems in 2007, Beijing Jiaotong University, China. Major field includes satellite communications and mobile communications.

He works as a researcher in Shenyang Ligong University, China, form 2007. His current research interests are the key technologies of Advanced Orbiting Systems and signal processing technologies in high speed mobile communication systems.

ZHANG Yan-qin, born in 1984, post graduate.

ZHANG Zi-jing, born in 1984, post graduate. 